Revista Med 26(1) 2018

\title{
EN RATAS, EL CONSUMO DE BEBIDAS NEGRAS, NORMALES O LIGHT, INCREMENTA EL PESO CORPORAL Y LA ANSIEDAD
}

\author{
LAURA LUCÍA ESLAVA-GONZÁLEZ¹, MABEL HENAO-PACHECO ${ }^{2}$, MARISOL ESCAÑO' ${ }^{1}$ ZULMA DUEÑAS², \\ ${ }^{1}$ Gimnasio Iragua. \\ ${ }^{2}$ Facultad de Medicina, Departamento de Ciencias Fisiológicas, Grupo de Neurobiología \\ y Comportamiento, Universidad Nacional de Colombia, Sede Bogotá.
}

Correspondencia: Zulma Dueñas, Carrera 30 \# 45-03, Edificio 471. Oficina 430

Tel: (571) 3165466, Bogotá, Colombia.zjduenasg@unal.edu.co

Recibido: 25 de agosto de 2017 Aceptado: 24 de octubre de 2017

\begin{abstract}
Resumen
El consumo de bebidas negras carbonatadas se asocia con obesidad debido a su alta concentración de azúcar. En efecto, la prevalencia de obesidad, sobre todo en niños, va en aumento, por lo que son pertinentes los estudios de factores de riesgo y los programas de prevención. En este sentido, el objetivo de este estudio piloto fue identificar, en un modelo animal, si el consumo de bebidas negras carbonatadas, normales y de dieta o light, afecta el peso y el comportamiento ansioso. Se seleccionaron 15 ratas Wistar macho, divididas en 3 grupos: un primer grupo de control, que bebía agua exclusivamente; un segundo grupo con suministro de agua y bebida negra carbonatada normal; un tercero con agua y bebida negra carbonatada light. Cada dos días se midió el consumo de bebida y alimento y cada semana se pesaron todos los sujetos. Pasadas cuatro semanas, se evaluaron comportamientos relacionados con ansiedad, utilizando el Laberinto en Cruz Elevado. Se encontraron diferencias significativas estadísticamente en el incremento del peso corporal de las ratas que consumieron bebidas negras normales y light, comparadas con el grupo que solo consumió agua. También se observó mayor consumo de las bebidas carbonatadas, si bien el consumo de alimento se mantuvo en proporciones muy similares para todos los grupos. Con respecto al tiempo de permanencia en los brazos cerrados, fue mayor en las ratas que consumieron bebida light comparadas con el grupo de control y, finalmente, ese mismo grupo mostró menor número de inmersiones de la cabeza en el laberinto. En conclusión, este estudio preliminar permite sugerir que el consumo de bebidas negras carbonatadas, normales y light, se asocia con un incremento del peso corporal de los animales y con mayor ansiedad.
\end{abstract}

Palabras clave: ansiedad; aspartame; peso corporal; ratas.

\section{IN RATS, CONSUMPTION OF BLACK DRINKS, NORMAL OR LIGHT, INCREASES CORPORAL WEIGHT AND ANXIETY}

\section{Summary}

The consumption of carbonated black beverages is associated with obesity due to their high concentration of sugar. In effect, the prevalence of obesity, especially in children, is increasing; therefore, studies of risk factors and prevention programs are relevant. In this sense, the objective of this pilot study was to identify, in an animal model, whether the consumption of normal and diet or light black carbonated beverages affects weight and anxious behaviors. Fifteen male Wistar rats were selected and divided into 3 groups: a first control group, which drank water exclusively; 
a second group which drank both water and a normal black carbonated beverage; a third group which drank both water and a light black carbonated beverage. Every two days their food and beverage consumption was measured, and every week all the subjects were weighed. After four weeks, behaviors related to anxiety were evaluated using the Elevated Plus Maze. Statistically significant differences were found, including the increase in body weight of the rats that consumed normal and light black beverages compared with the group that only consumed water. There was also a higher consumption of carbonated beverages, although the consumption of food remained very similar for all groups. Regarding the time spent in the closed arms, it was greater for the rats that consumed light drinks compared with the control group; and finally, that same group showed a lower number of head dips (dipping) in the maze. In conclusion, this preliminary study suggests that the consumption of normal and light black carbonated beverages is associated with an increase in the body weight of animals and an increase in anxiety.

Keywords: anxiety; aspartame; corporal weight; rats.

\section{EM RATOS, O CONSUMO DE BEBIDAS NEGRAS, NORMAIS OU LIGHT, INCREMENTA O PESO CORPORAL E A ANSIEDADE}

\section{Resumo}

O consumo de bebidas negras gaseificadas se associa com obesidade devido a sua alta concentração de açúcar. Em efeito, a prevalência de obesidade, sobre tudo em crianças, vai em aumento, o que torna pertinentes os estudos de fatores de risco e os programas de prevenção. Neste sentido, o objetivo deste estudo piloto foi identificar, em um modelo animal, se o consumo de bebidas negras gaseificadas, normais e de dieta ou light, afeta o peso e o comportamento ansioso. Se selecionaram 15 ratos Wistar macho, divididos em 3 grupos: um primeiro grupo de controle, que bebia água exclusivamente; um segundo grupo com fornecimento de água e bebida negra gaseificada normal; um terceiro com água e bebida negra gaseificada light. A cada dois dias mediu-se o consumo de bebida e alimento e a cada semana se pesaram todos os sujeitos. Após quatro semanas, foram avaliados os comportamentos relacionados com ansiedade, utilizando o Labirinto em Cruz Elevado. Encontraram-se diferenças significativas estatisticamente no aumento do peso corporal dos ratos que consumiram bebidas negras normais e light, comparadas com o grupo que consumiu somente água. Também se observou um maior consumo das bebidas gaseificadas, embora o consumo de alimento tenha se mantido em proporções muito similares para todos os grupos. Com respeito ao tempo de permanência nos braços cerrados, foi superior nos ratos que consumiram bebida light comparadas com o grupo de controle e, finalmente, esse mesmo grupo mostrou um número inferior de imersões da cabeça não labirinto. Em conclusão, este estudo preliminar permite sugerir que o consumo de bebidas negras gaseificadas, normais e light, está associado com um aumento do peso corporal dos animais e com um maior nível de ansiedade.

Palavras-chave: ansiedade; aspartame; peso corporal; ratos.

\section{Introducción}

La bebida negra carbonatada fue inventada por el farmacéutico Jhon Pemberton en mayo de 1886 (1). Su composición incluye agua, azúcar, dióxido de carbono, color caramelo, acidulantes, saborizantes y cafeína (2) y vale mencionar que actualmente es la gaseosa más vendida en todo el mundo $(3,4)$. Además, en los últimos 30 años, el consumo de esta bebida se ha triplicado en población infantil y adolescente, lo que hace que esta bebida repre- sente cerca del $56 \%$ del total de la ingesta de líquidos consumidos por los infantes, seguida por la leche con $18 \%$ y el agua con un $9 \%$ (3). Diversos estudios han demostrado que la obesidad y el consumo de la bebida negra carbonatada están íntimamente ligados (5-8). Además, se ha evidenciado la relación entre el consumo de esta bebida con enfermedades como la osteoporosis (9) y la degradación de la salud dental (10). 
Una lata normal de $330 \mathrm{ml}$ contiene aproximadamente $32 \mathrm{mg}$ de cafeína, mientras que una lata de bebida negra carbonatada light tiene $42 \mathrm{mg}$ (4). Se sabe que la cafeína es antagonista de los receptores de adenosina en el Sistema Nervioso Central (11). La adenosina es un neuromodulador que tiene efectos sedantes, hipnóticos y ansiolíticos (tranquilizantes), entre otros $(12,13)$. Como antagonista de estos receptores, la cafeína bloquea su acción $y$, por tanto, inhibe sus efectos sedativos $e$ inductores de sueño. Tales efectos ansiogénicos, a dosis moderadas y altas, de la cafeína han sido comprobados en la mayoría de los estudios farmacológicos $(12,13)$.

Ahora bien, debido al rápido ingreso del azúcar al torrente sanguíneo, se inducen fluctuaciones rápidas en los niveles de glucemia y, por lo tanto, el individuo incrementa sus niveles energéticos. Este hecho está demostrado por una cantidad significativamente alta de estudios, en los cuales queda clara la relación entre el azúcar y la hiperactividad $(14,15)$.

En el caso de las bebidas etiquetadas como light, el azúcar ha sido reemplazado por endulzantes no calóricos como el aspartame y el acetasulfame $\mathrm{k}$. A las personas obesas se les sugiere reducir al mínimo el consumo de gaseosa y que cuando de todas formas lo hagan, consuman de preferencia bebidas con esta etiqueta. Sin embargo, no se ha determinado si la sustitución de la bebida negra carbonatada normal por la debida de dieta o light contribuye a disminuir el peso corporal y la ansiedad.

Por lo anterior, se planteó la posibilidad de responder si tal recomendación tiene un sustento demostrable. Para ello, se planteó un estudio con un modelo animal. Se trata de un estudio piloto llevado a cabo con ratas de laboratorio de la cepa Wistar a las que se dio la posibilidad de consumir bebida carbonatada normal, light y agua. Los sujetos del experimento se compararon con un grupo de control que solo consumía agua. Se midió el consumo de agua y comida cada dos días y el peso fue calculado semanalmente. Las mediciones de ansiedad se hicieron según el método del laberinto en cruz elevado (LCE), instrumento ampliamente validado y utilizado para evaluar este propósito (16).

\section{Objetivo}

El objetivo de este estudio piloto fue identificar, en un modelo animal con ratas, si el consumo de bebida negra carbonatada, light o normal, afecta el peso y el comportamiento ansioso en los sujetos del experimento.

\section{Materiales y métodos}

\section{Animales}

Se utilizaron 15 ratas macho de la cepa Wistar, de 35 días de nacidas, provenientes del Bioterio de la facultad de Medicina Veterinaria de la Universidad Nacional. Las ratas se mantuvieron bajo condiciones de temperatura $y$ humedad controladas.

\section{Ingesta de bebidas}

$\mathrm{Al}$ inicio, las ratas se pesaron y dividieron en 3 grupos de 5 ratas cada uno. A todos los grupos se les proporcionaba $250 \mathrm{~g}$ de alimento cada dos días. El grupo 1, de control, tenía de bebida solo agua $(500 \mathrm{ml})$. El grupo 2 tenía $500 \mathrm{ml}$ de agua y una botella con $500 \mathrm{ml}$ de bebida carbonatada normal. El grupo 3 tenía $500 \mathrm{ml}$ de agua y $500 \mathrm{ml}$ de bebida negra carbonatada light. Cada dos días se ajustaban las dosis de comida y bebidas.

El cambio de camas se hizo cada vez que se consideró necesario, por aspecto y olor. Todos los procedimientos llevados a cabo fueron realizados de conformidad con las normas éticas y legales exigidas para la investigación con animales de laboratorio en Colombia (17).

El experimento duro 4 semanas, durante las cuales se midió el consumo de líquidos cada dos días y se llenaron nuevamente las botellas. Se procedió de igual manera con el alimento sólido.

Se hicieron evaluaciones de peso semanalmente. Al día siguiente de terminado el protocolo de consumo de bebidas negras, se hicieron las pruebas en el LCE.

\section{Prueba Comportamental}

El LEC es una prueba comportamental ampliamente utilizada que se fundamentada en la aversión natural de roedores por la altura y los espacios abiertos (16). Consiste en una plataforma acrílica de color negro en forma de cruz que tiene dos brazos abiertos (sin paredes laterales) de $50 \times 10 \mathrm{~cm}$ y dos cerrados de $50 \times 10$ con paredes de $40 \mathrm{~cm}$ de altura. El instrumento está elevado a una altura de $50 \mathrm{~cm}$ del suelo y es descubierto en la parte superior (16) (figura 1).

En cada sesión se puso al animal en el centro frente a uno de los brazos abiertos y se permitió que explorara durante cinco minutos. Los comportamientos fueron grabados por medio de una cámara de video, ubicada en 


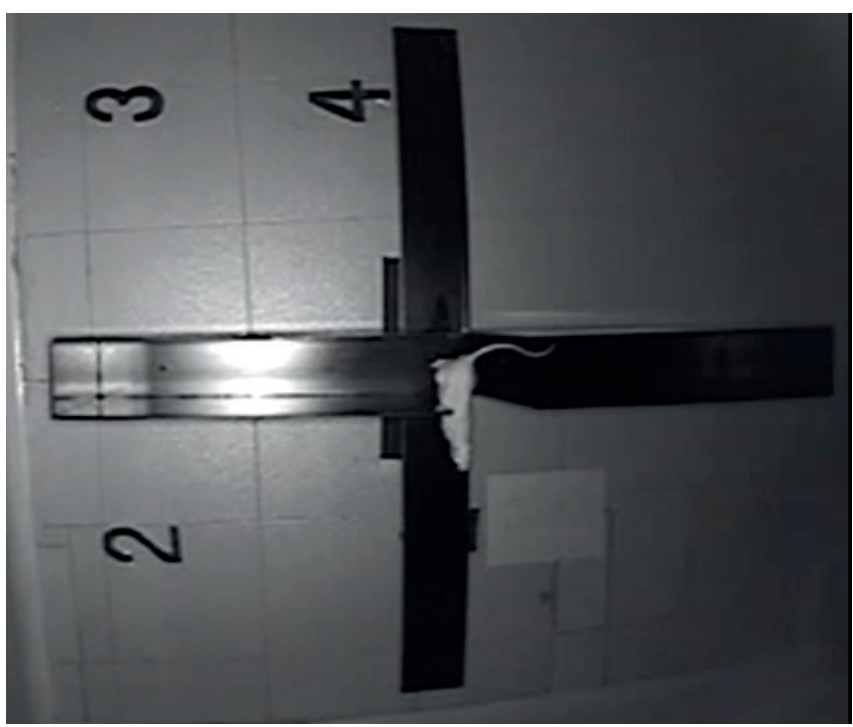

Figura 1. Imagen de una rata en el laberinto en cruz elevado.

el techo de la habitación. La codificación de los comportamientos se realizó por medio del programa X-ploRat 2005. Se registraron los patrones comportamentales de interés que para el caso correspondieron al tiempo de permanencia en los brazos cerrados y el número de inmersiones o hundimientos de la cabeza (head dipping). Se consideró que un animal había entrado a un brazo cuando pisó con las cuatro patas dicho brazo y se consideró inmersión de la cabeza, cada vez que la rata exploró por fuera del laberinto sumergiendo su cabeza en el vacío. El análisis estadístico se hizo utilizando la prueba de Análisis de varianza de un factor ANOVA y se estableció como diferencia significativa cuando el valor $\mathrm{p}$ fue menor de 0,05 .

\section{Resultados}

Los resultados que aquí se presentan son una primera aproximación al efecto que tiene el consumo de bebida negra carbonatada normal y light sobre el incremento del peso corporal y la ansiedad. En primer lugar, se destaca que los dos grupos experimentales, es decir los que tenían la opción de consumir bebida negra, tuvieron preferencia por esta bebida y no por el agua normal. En la figura 2 se puede observar el volumen medido en las botellas correspondientes a agua o bebida negra, en los dos grupos experimentales y solo agua en el grupo de control (a). Efectivamente, se identifica que los grupos que, teniendo la opción de tomar agua o bebida negra, con el tiempo incrementaban la ingesta de la bebida negra. Sin embargo, el consumo de alimento sólido se mantuvo igual para todos los grupos.

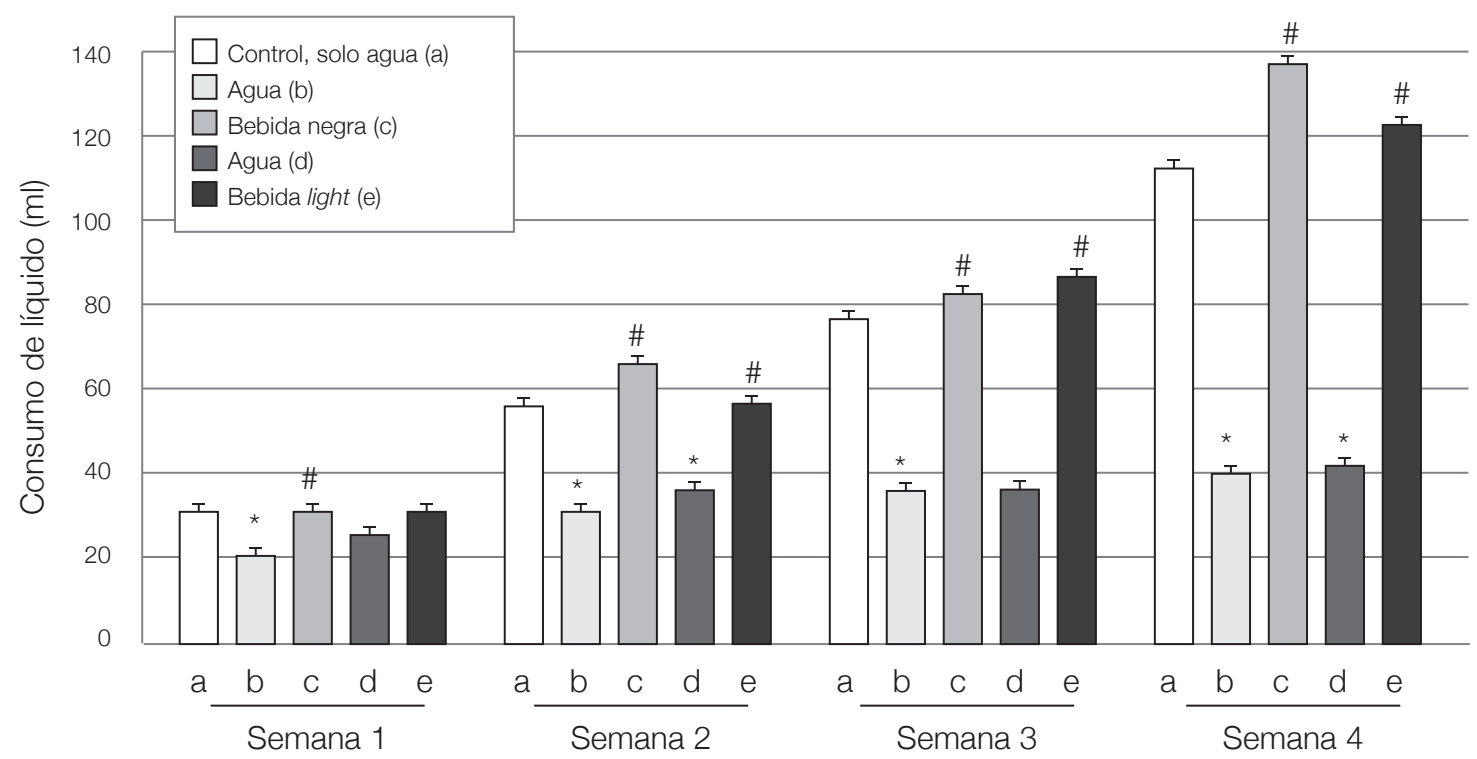

Figura 2. Consumo de líquido. Consumo de líquidos diario, promediado por semana en cada grupo experimental. Los grupos se identifican como a) Control solo con agua, b) y c) experimental agua y bebida negra normal, d) y e) agua y bebida negra light. A NOVA de un factor, $n=5, * p<0,05$ comparado con el grupo control que solo bebe agua; $\# p<0,05$ comparado con la cantidad de agua bebida frenta a la ingesta de bebida negra. 
Con respecto al peso corporal, es importante anotar que, como era de esperarse por condiciones de crecimiento, todos los grupos incrementaron paulatinamente su peso (figura 3). Sin embargo, los grupos que consumieron bebidas negras tuvieron mayor aumento de peso, comparado con el grupo que solo tenía agua y comida. En la figura 3 se puede observar que la diferencia es aún mayor en el grupo que consumió bebida light, si bien los dos grupos que ingerían bebida carbonatada obtuvieron el mismo peso al final del experimento.

En el laberinto en cruz elevado se registraron los comportamientos relacionados con la permanencia en los

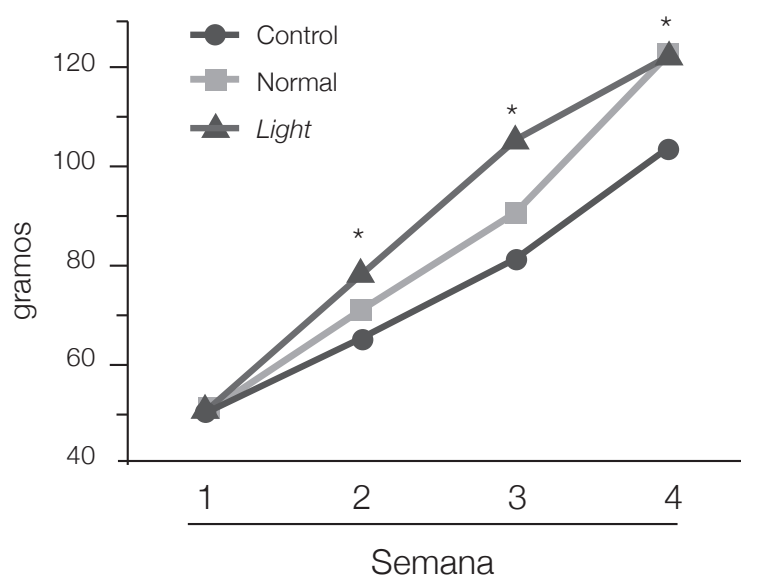

Figura 3. Registro del peso corporal de las ratas de los tres grupos. Se grafican los promedios del peso al finalizar cada semana del estudio. ${ }^{*} p<0,05$ comparado con el grupo control (CTRL).

brazos cerrados. La figura 4 muestra el tiempo de permanencia de las ratas en los brazos cerrados del laberinto. Se puede observar una tendencia del grupo que tuvo la opción de consumir bebida negra normal a permanecer más tiempo en espacios cerrados y una diferencia estadísticamente significativa en el grupo que consumió bebida light.

El último de los comportamientos medidos correspondió a la inmersión o hundimiento de la cabeza que, si bien no es un comportamiento que refleje directamente el estado ansioso de los animales, permite identificar qué tanto exploran si están en un espacio que les da la posibilidad de identificar "vacío". El único grupo que mostró diferencias fue el que consumió bebida light, que tuvo una marcada

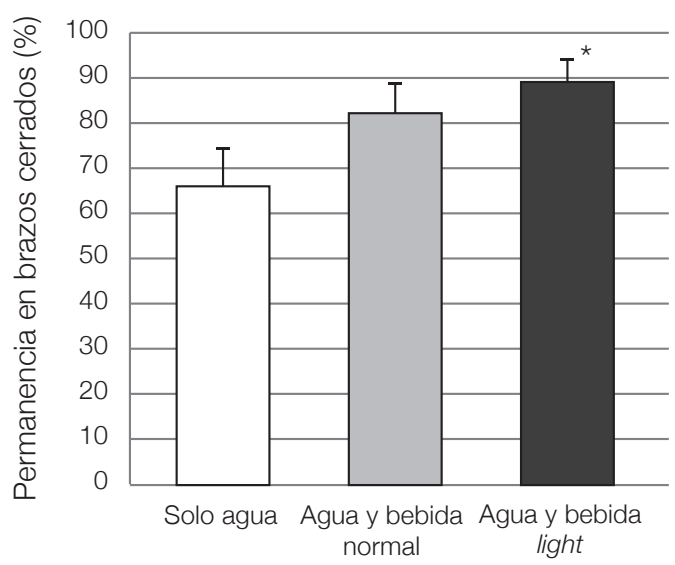

Figura 4. Tiempo de permanencia en los brazos cerrados del laberinto.

disminución de este comportamiento, comparado con los otros dos grupos (figura 5).

\section{Discusión}

La obesidad en Colombia, como en el mundo, ha aumentado y particularmente se ha visto un mayor número de niños y adolescentes con este padecimiento. La industria de las bebidas azucaradas, por su parte, ha buscado estrategias de producción y mercadeo que mantengan el alto consumo de sus productos. Dentro de dichas estrategias, ha implementado suplir el azúcar por edulcorantes, promocionando entonces sus productos como light o de dieta. Los resultados que aquí se presentaron

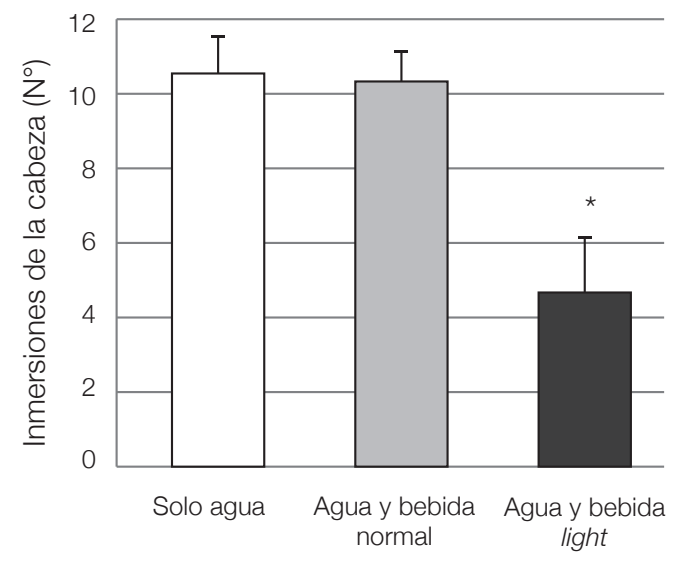

Figura 5. Número de inmersiones de la cabeza $(* p<0,05, n=5)$. 
evidencian que, sin importar si la bebida es endulzada con azúcar normal o con un edulcorante diferente, hay aumento tanto en la ingesta de la bebida, como en el peso corporal en los consumidores, en este caso ratas. De hecho, hay estudios que demuestran que al consumir edulcorantes no nutritivos como el aspartame y acesulfame k, se aumenta el apetito (19-22) y el consumo de alimentos debido a que, al consumir estos endulzantes, no se estimularía la liberación de insulina y leptina $(22,23)$ y en consecuencia no se produce saciedad incrementando indirectamente el consumo calórico al haber una mayor necesidad de comer (20-25). En ese caso, el incremento de peso en las ratas consumidoras de bebida negra carbonatada normal pudo deberse a la alta ingesta de azúcar. Sin embargo, no es claro el motivo por el cual las ratas que consumieron bebida light (no calórica), aumentaron de peso, dado que en promedio todos los grupos mantenían la cantidad de alimento solido consumido de manera muy similar. Existen algunos reportes en la literatura, como el de Polák y sus colaboradores (21), quienes demostraron que el consumo en ratones de edulcorantes resultó en un incremento significativo del peso corporal, aunque sin variaciones en el consumo de alimento sólido. Por otra parte, un estudio más reciente señala que hay enorme controversia en cuanto al beneficio del consumo de edulcorantes no nutritivos, con respecto a los beneficios y riesgos potenciales que pueda conllevar su uso prolongado (22). Es importante anotar que existen reportes que muestran una asociación entre el consumo de estas bebidas y problemas metabólicos $(26,27)$. Por ejemplo, en la revisión hecha por Romo-Romo y sus colaboradores (28) señala que algunos estudios observacionales sugieren una asociación entre el consumo de edulcorantes no nutritivos y el desarrollo de enfermedades metabólicas, si bien es muy importante realizar más estudios con grupos homogéneos y en los que se evalúen variables como la adiposidad. En este mismo sentido, otra revisión publicada en 2016 (29), señala que se ha encontrado un aumento en el peso corporal y el riesgo cardiometabólico en personas consumidoras de bebidas endulzadas con edulcorantes no calóricos. Si bien no hay una asociación causal directa, señala que hay mucha congruencia en las investigaciones con animales $y$ los hallazgos observacionales con seres humanos, por lo que preocupa que se siga incrementando el consumo de edulcorantes (29).

Con respecto a los efectos sobre la ansiedad, evaluada en el LCE, se evidenció un incremento en la permanencia de los sujetos en los brazos cerrados del laberinto. $\mathrm{Si}$ bien es cierto, la tendencia natural de los roedores es permanecer en espacios cerrados, el notorio incremento de permanencia señala un aumento de la ansiedad en las ratas que consumieron bebidas negras carbonatadas. Aunque es de advertir que no se han hecho estudios que relacionen el efecto del consumo de bebidas negras carbonatadas con la ansiedad. Aun así, este efecto podría atribuirse a la cafeína, que es un antagonista no selectivo de los receptores de adenosina y tiene efectos neuromoduladores (13), pues, al bloquear su acción, se bloquearían también los efectos sedativos o ansiolíticos controlados por estos receptores. Esto explicaría el que las ratas se muestren más ansiosas $(11,13,30)$.

A pesar de lo anterior, el comportamiento de agachar la cabeza, que podría ser un indicador indirecto de ansiedad, disminuyó en el grupo que consumió bebida light. Este comportamiento también puede denotar que el animal está evaluando o explorando el entorno, lo que podría estar asociado a esa conducta.

Es importante mencionar que, para este estudio, se utilizaron solamente ratas varones, con el fin de evitar respuestas hormonales naturales de las hembras que pudieran o enmascarar o bien potenciar el consumo de bebidas y los comportamientos.

Finalmente, es de advertir que este es un ensayo piloto y preliminar, que corresponde a una primera aproximación, en el que solo se evaluaron 5 animales por grupo. Sin embargo, en el estudio se evidencia un incremento de peso asociado a la ingesta de una bebida light, lo que permite llamar la atención sobre la necesidad de regular su consumo, sobre todo en personas obesas, quienes utilizan edulcorantes como parte de su dieta y, más aún, en niños a quienes cada vez más se suministra bebidas con edulcorantes.

Conflicto de intereses: ninguno.

Financiación: este proyecto fue financiado por la Facultad de Medicina y la Dirección de Investigación de la Sede Bogotá, Código Hermes 28075.

\section{Referencias}

1. Castro Soto Gustavo., Coca-Cola La historia negra de las aguas negras, primera Parte. CIEPAC. 2015. Disponible en: http://www.rebelion.org/docs/10924.pdf

2. Espinoza D, Marcela Lissette, Pérez-Sánchez ML, Antonio H. Diseño del sistema de tratamiento de agua del enjuague 
final en una embotelladora, Universidad de Guayaquil. Facultad Ingeniería Química, 2011. Consultado: agosto de 2017. Disponible en http://repositorio.ug.edu.ec/handle/redug/4645

3. Muñoz Ana. Las marcas más vendidas del mundo. 2016. Consultado: agosto 2017. Disponible en: http://www. ticbeat.com/cyborgcultura/estas-son-las-marcas-mas-vendidas-del-mundo-y-no-te-las-esperas/

4. Gaille Brandon. 25 Great Coca Cola Sales Statistics. Consultado: agosto de 2017. Dispobible en http://brandongaille.com/24-great-coca-cola-sales-statistics/

5. Rippe JM, Angelopoulos TJ. Relationship between Added Sugars Consumption and Chronic Disease Risk Factors: Current Understanding. Nutrients. 2016 Nov 4; 8(11).

6. Celec P, Behuliak M. Behavioural and endocrine effects of chronic cola intake. J Psychopharmacol. December 2010; 10: 1569-72.

7. Hu Y, Costenbader KH, Gao X, Al-Daabil M, Sparks JA, Solomon DH, Hu FB, Karlson EW, Lu B. Sugar-sweetened soda consumption and risk of developing rheumatoid arthritis in women. Am J Clin Nutr. 2014; 100(3): 959-67.

8. Gómez-Miranda, L. M., Jiménez-Cruz, A., \& Bacardi-Gascón, M. Estudios aleatorizados sobre el efecto del consumo de bebidas azucaradas sobre la adiposidad en adolescentes y adultos: revisión sistemática. Nutrición Hospitalaria. 2013; 28(6): 1792-1796.

9. Murillo-Godínez, G. Ingesta de refrescos-de cola-y riesgo de osteoporosis. Consultado: agosto de 2017; disponible en: http://www.portalesmedicos.com/publicaciones/articles/4490/1/Ingesta-de-refrescos-de-cola-y-riesgo-deosteoporosis.html

10. Buttani, N., Riveros, E., \& Dolonguevich, E. Cambios morfológicos en la estructura del esmalte dental debido al consumo de bebidas industrializadas. Revista de la Facultad de Odontología. Universidad Nacional de Cuyo. 2015: 9(1): 21-3.

11. Cunha Rodrigo A. Caffeine, adenosine receptors, memory and Alzheimer disease. Medicina Clínica. 2008; 131(20): 790-5.

12. Contreras M, E. Adenosina: acciones fisiológicas y farmacológicas. Arch. Biol. Med. Exp. 1990; (23): 1-12.

13. Kazemzadeh-Narbat M, Annabi N, Tamayol A, Oklu R, Ghanem A, Khademhosseini A. Adenosine-associated delivery systems. J Drug Target. 2015; 23(7-8): 580-96.

14. Yu C-J, Du J-C, Chiou H-C, et al. Sugar-Sweetened Beverage Consumption Is Adversely Associated with Childhood Attention Deficit/Hyperactivity Disorder. Tchounwou PB, ed. International Journal of Environmental Research and Public Health. 2016; 13(7): 678.

15. Kim Y, Chang H. Correlation between attention deficit hyperactivity disorder and sugar consumption, quality of diet, and dietary behavior in school children. Nutrition Research and Practice. 2011; 5(3): 236-45.

16. Walf AA, Frye CA. The use of the elevated plus maze as an assay of anxiety-related behavior in rodents. Nature protocols. 2007; 2(2): 322-8.
17. Resolución 8430 de 1993 y Ley 84 de 1989. Consultado: agosto de 2017, disponible en: https://www.minsalud. gov.co/Normatividad_Nuevo/RESOLUCION\% 20 8430\%20DE\%201993.pdf

18. Riveros-Barrera Irene, Dueñas Zulma. Efecto de la alopregnanolona sobre la ansiedad en ratas con separación materna durante la lactancia. Rev. Fac. Med. 2014; 62(2): 229.

19. Durán, S., Cordón, K., \& Rodríguez, M. D. P. Edulcorantes no nutritivos, riesgos, apetito y ganancia de peso. Revista chilena de nutrición. 2013; 40(3): 309-14.

20. Durán Agüero, S., Blanco Batten, E., Rodríguez Noel, M. D. P., Cordón Arrivillaga, K., Salazar de Ariza, J., Record Cornwall, J., \& Encina Vega, C. Asociación entre edulcorantes no nutritivos y riesgo de obesidad en estudiantes universitarios de Latinoamérica. Revista médica de Chile, 2015; 143(3): 367-73.

21. Polyák E, Gombos K, Hajnal B, Bonyár-Müller K, Szabó S, Gubicskó-Kisbenedek A, Marton K, Ember I. Effects of artificial sweeteners on body weight, food and drink intake. Acta Physiol Hung. 2010; 97(4): 401-7.

22. Sharma A, Amarnath S, Thulasimani M, Ramaswamy S. Artificial sweeteners as a sugar substitute: Are they really safe? Indian J Pharmacol. 2016; 48(3): 237-40.

23. Glendinning JI, Elson AET, Kalik S, et al. Taste Responsiveness to Sweeteners Is Resistant to Elevations in Plasma Leptin. Chemical Senses. 2015; 40(4): 223-31.

24. Fowler SP. Low-calorie sweetener use and energy balance: Results from experimental studies in animals, and largescale prospective studies in humans. Physiol Behav. 2016; 164(Pt B).

25. Flores Cotrina, L. I., \& Romero Lazo, C. L. Niveles de insulina post ingesta de edulcorantes en adultos sanos. Nutrición, Tesis. 2014. Lima Perú Disponible en http:// cybertesis.unmsm.edu.pe/handle/cybertesis/3761

26. Yang Q. Gain weight by "going diet?" Artificial sweeteners and the neurobiology of sugar cravings: Neuroscience 2010. The Yale Journal of Biology and Medicine. 2010; 83(2): 101-8.

27. Ylvetsky A, Rother KI, Brown R. Artificial sweetener use among children: epidemiology, recommendations, metabolic outcomes, and future directions. Pediatric clinics of North America. 2011; 58(6): 1467-1480.

28. Romo-Romo A, Aguilar-Salinas CA, Brito-Córdova GX, Gómez Díaz RA, Vilchis Valentín D, Almeda-Valdes P. Effects of the Non-Nutritive Sweeteners on Glucose Metabolism and Appetite Regulating Hormones: Systematic Review of Observational Prospective Studies and Clinical Trials. PLoS One. 2016; 11(8): e0161264

29. Azad MB, Abou-Setta AM, Chauhan BF, Rabbani R, Lys J, Copstein L, Mann A et al. Nonnutritive sweeteners and cardiometabolic health: a systematic review and meta-analysis of randomized controlled trials and prospective cohort studies. CMAJ. 2017; 189 (28): E929-39.

30. Richards G, Smith A. Caffeine consumption and self-assessed stress, anxiety, and depression in secondary school children. J Psychopharmacol. 2015; 29 (12): 1236-47. 